\title{
Age-of-acquisition, imagery, familiarity and meaningfulness norms for 543 words
}

\author{
R. PAUL STRATTON, KENNETH A. JACOBUS, and BONNIE BRINLEY \\ University of Kentucky, Lexington, Kentucky 40506
}

\begin{abstract}
The age at which words are first learned appears to be more influential in determining the ease of retrieving words from semantic memory than objective frequency, familiarity, imagery, and meaningfulness. To facilitate research on a wider variety of tasks, we present norms for 543 words for age-of-acquisition, imagery, familiarity, and meaningfulness. Most of the words form single-solution anagraris. There are 471 six-letter nouns and 72 five-letter words. Also reported are the means, SDs, and ranges for each dimension and the intercorrelations between dimensions. Intergroup reliabilities ranged from .847 to .982 .
\end{abstract}

Recent studies have indicated that the age at which words are first learned is influential in determining the ease of retrieving words from semantic memory. Frequency of usage during childhood was found to predict latency to name category instances more accurately than adult frequency of usage (Loftus \& Suppes, 1972). Age-of-acquisition as rated by young adults was found by Carroll and White (1973) to be a more relevant variable than objective frequency in predicting latency to name pictures. Rated age-of-acquisition also predicted the speed and likelihood of solving anagrams more accurately than rated familiarity, objective frequency, imagery, and meaningfulness (Stratton, Jacobus, \& Brinley, Note 1).

The norms reported in this paper provide adult norms on rated age-of-acquisition, rated imagery, rated familiarity, and meaningfulness for 543 words. Two word samples from these norms were used in an earlier study (see Note 1).

\section{METHOD}

\section{Subjects}

A total of 879 male and female volunteers from introductory psychology classes contributed to the six-letter word norms during the 1973 fall semester and the 1974 spring semester. An additional 204 males and females from the same subject pool contributed to the five-letter norms during the 1974 summer and fall semesters. Different subjects participated in each segment of the study.

\section{Words}

The 471 six-letter words were selected from Thorndike and Lorge (1944) and included every six-letter noun which occurred at least once per million words, began with a consonant, had no repeated letters, and appeared to be the only word which could be formed with those letters. Some exceptions were later discovered (e.g., thread-hatred) but were included here for

The collection of the norms was made possible in part by grants from the University of Kentucky Research Foundation and in part by the generous assistance of Joe Prus and Chris and in part by the generous assistance of Joe Prus and Chris Department of Psychology, University of Kentucky, Lexington, Kentucky 40506. possible use in tasks other than anagrams. The 72 tive-letter words form single-solution anagrams and had normative anagram-solving latency data available (Tresselt \& Mayzner, 1966). Some of these words begin with a vowel and have repeated letters. Thorndike and Lorge (1944) was also the original source for the five-letter word sample, although several words have frequencies of less than one per million.

\section{Procedure}

The general procedure for the age-of-acquisition, imagery, and familiarity ratings was to present all of the words to each subject in a booklet with approximately an equal number of words per page. Subjects rating five-letter words also rated 50 randomly selected six-letter words. The order of the pages was counter-balanced across subjects. Ratings were obtained from subjects in small groups and recorded on answer sheets which were later machine scored. Subjects completed half of the ratings in each of two 1-h sessions and were encouraged to use as much time as necessary. For the ratings of six-letter words, at least 50 subjects responded to each word and at least 40 for the five-letter words.

The specific procedures for the rating tasks were as follows. Age-of-acquisition ratings were obtained using the Carroll and White (1973) instructions. The scale ranged from 1 (prenursery, age 2) to 9 (eighth grade and above, age 13 and older). Imagery ratings were obtained by using the procedures and instructions given by Paivio, Yuille, and Madigan (1968), with the exception that a five-point, rather than a seven-point, scale was used. The ease or speed of arousing a sensory image was rated with 1 defined as "very difficult, image aroused after a long delay or not at all." A 5 was defined as "very easy, image aroused immediately." Familiarity ratings provided an index of contemporary usage for our adult subject population. Noble's (1953) rating scale ( 1 = "never used," $5=$ "used every day") and instructions were used.

Meaningfulness was defined as the mean number of associations given under Noble's (1952) instructions and scoring procedures for a $30-\mathrm{sec}$ production period. The subjects were given booklets of 25 words with each word repeated 15 times per page. Orders of presentation were randomized across subjects. Responses were obtained from at least 30 subjects per word for both sets of words.

\section{RESULTS AND DISCUSSION}

The words are listed in the Appendix along with each word's mean and SD on each dimension. The summary 
statistics and intercorrelations are given in Table 1. The words represent a wide range of values along each dimension. The intergroup reliability was obtained by randomly halving the subject sample and correlating the obtained mean scores between samples. As indicated in Table 1, intergroup reliabilities ranged from .847 to 982. The 50 six-letter words which were rated a second time with the five-letter words provide an estimate of test-retest reliability. The obtained correlations between word mean scores on the dimensions are given in Table 1 and ranged from .896 to .968 . In addition, 61 of the words were also in Paivio et al. (1968), and the correlations between the two sets of norms were .897 for imagery and .709 for meaningfulness. The Paivio et al. (1968) words have also been rated for age-of-acquisition (Jacobus \& Stratton, Note 2), and the correlation for the 61 words contained in both sets of norms was .965 .

Table 1 shows that the variables are highly correlated, although these correlations are not as high as would be expected if the scales measured the same underlying dimension. The correlation of .72 between imagery and meaningfulness corresponds to the correlation of .72 found by Paivio et al. (1968), reflecting the stability of the scaling procedures. Most importantly, although age-of-acquisition is highly correlated with the other variables, it has been found to have an independent effect on the retrieval of words from semantic memory (Stratton, et al., Note 1).

For anagram studies, some words have been found to be double-solution words in that other words may be formed from the same letters. We are aware of the following double-solution words, although ingenious undergraduates will undoubtedly provide additional ones in the future (an asterisk designates words in the norms); clause*-sacule, copula-cupola*, damsel*-medals, danger*-gander*-garden*, downer-wonder*, filter*-lifter, hatred ${ }^{*}-$ thread ${ }^{*}-$ dearth, mante ${ }^{*}$-mantle, pathos*-potash*, retain-retina*, shiner-shrine*, and shriek*-shrike*.

\section{REFERENCES}

Carroll, J. B., \& White, M. R. Age-of-acquisition norms for 220 picturable nouns. Journal of Verbal Learning and Verbal Behavior, $1973,12,563-576$.

Loftus, E. F., \& Suppes, P. Structural variables that determine the speed of retrieving words from long-term memory. Journal of Verbal Learning and Verbal Behavior, 1972, 11, 770-777.

Noble, C. E. An analysis of meaning. Psychological Review, $1952,59,421-430$.

Noble, C. E. The meaning-familiarity relationship. Psychological Review, 1953, 60, 89-98.

Paivio, A., Yuille, J. C., \& Madigan, S. A. Concreteness, imagery and meaningfulness values for 925 nouns. Journal of Experimental Psychology Monograph Supplements, 1968, 76, (1, Part 2)

Thorndike, E. L., \& Lorge, 1.The teacher's word book of 30,000 words. New York: Teachers College Press, 1944.

Tresselt, M. E., \& Mayzner, M. S. Normative solution times for a sample of 134 solution words and 378 associated anagrams. Psychonomic Monograph Supplements, 1966, 1, 293-298.
Table 1

Correlations Among Word Characteristics

\begin{tabular}{lcccc}
\hline Word Characteristic & 1 & 2 & 3 & 4 \\
\hline 1. Age of Acquisition & & & & \\
2. Imagery & -.68 & & & \\
3. Familiarity & -.74 & .45 & & \\
4. Meaningfulness & -.70 & .72 & .71 & \\
Mean & 6.38 & 3.21 & 3.31 & 4.48 \\
SD & 1.54 & 1.00 & .72 & 1.23 \\
Range & $1.54-$ & $1.14-$ & $1.43-$ & $.35-$ \\
& 8.90 & 5.00 & 4.84 & 7.42 \\
Intergroup Reliability & .982 & .939 & .952 & .847 \\
Test-Retest Reliability & .968 & .921 & .896 & .900 \\
\hline
\end{tabular}

\section{REFERENCE NOTES}

1. Stratton, R. P., Jacobus, K. A., \& Brinley, B. Characteristics of nouns which determine ease of solving anagrams. Manuscript submitted for publication, 1974.

2. Jacobus, K. A., \& Stratton, R. P. Age-of-acquisition ratings for Pavio's 928 nouns. (Available from Kenneth A. Jacobus, Psychology Department, University of Kentucky, Lexington, Kentucky 40506).

(Received for publication November 4, 1974; accepted December $4,1974$.

\section{APPENDIX}

Listed on the following pages in alphabetical order are the 543 words together with each word's mean score and standard deviation for age of acquisition, imagery, familiarity, and meaningfulness.

\begin{tabular}{|c|c|c|c|c|c|c|c|c|}
\hline \multirow[b]{2}{*}{ Word } & \multicolumn{2}{|c|}{$\begin{array}{c}\text { Age of } \\
\text { Acquisition }\end{array}$} & \multicolumn{2}{|c|}{ Imagery } & \multicolumn{2}{|c|}{ Familiarity } & \multicolumn{2}{|c|}{$\begin{array}{c}\text { Mean- } \\
\text { ingfulness }\end{array}$} \\
\hline & Mean & SD & Mean & SD & Mean & SD & Mean & $\mathrm{SD}$ \\
\hline Adopt & 6.85 & 1.33 & 1.79 & 1.41 & 3.13 & .88 & 3.71 & 2.24 \\
\hline Agile & 7.92 & .93 & 1.92 & 1.45 & 3.13 & .90 & 4.28 & 1.60 \\
\hline Apron & 3.15 & 1.55 & 4.36 & 1.45 & 2.80 & 1.03 & 5.00 & 1.88 \\
\hline Audit & 8.69 & .67 & 1.71 & 1.48 & 3.20 & 1.08 & 4.21 & 2.08 \\
\hline Bacon & 2.62 & 1.30 & 4.93 & 1.33 & 4.60 & .55 & 6.07 & 1.82 \\
\hline Banker & 4.89 & 1.03 & 4.08 & 1.13 & 4.09 & .91 & 4.55 & 1.43 \\
\hline Banter & 8.33 & 1.06 & 1.98 & 1.42 & 2.41 & 1.01 & 2.07 & 1.82 \\
\hline Barony & 7.37 & 1.16 & 1.56 & 1.23 & 1.69 & .63 & 1.48 & 2.32 \\
\hline Basket & 4.23 & 1.41 & 4.56 & .93 & 4.10 & .70 & 4.85 & 2.16 \\
\hline Batch & 5.15 & 1.59 & 2.35 & 1.27 & 3.20 & 1.01 & 3.28 & 1.48 \\
\hline Baton & 6.15 & 1.54 & 4.14 & 1.41 & 2.27 & 1.03 & 3.71 & 2.02 \\
\hline Beach & 4.77 & 1.63 & 4.93 & 1.16 & 3.33 & .73 & 7.07 & 2.43 \\
\hline Beauty & 4.74 & 1.23 & 3.50 & 1.50 & 4.38 & .76 & 4.50 & 1.56 \\
\hline Bedlam & 8.02 & 1.17 & 2.56 & 1.52 & 2.80 & 1.08 & 3.59 & 2.19 \\
\hline Belfry & 8.10 & 1.14 & 2.88 & 1.65 & 2.50 & .91 & 4.21 & 2.70 \\
\hline Birth & 4.00 & 1.46 & 3.71 & 1.19 & 3.67 & .88 & 5.14 & 1.80 \\
\hline Bishop & 6.52 & 1.38 & 4.07 & 1.16 & 3.26 & .89 & 5.46 & 2.01 \\
\hline Blare & 7.46 & 1.16 & 1.93 & 1.41 & 2.67 & 1.04 & 3.92 & 1.87 \\
\hline Bleach & 5.66 & 1.38 & 3.61 & 1.44 & 3.78 & .80 & 5.72 & 2.65 \\
\hline Blight & 7.60 & 1.30 & 2.47 & 1.37 & 2.69 & .82 & 3.25 & 2.19 \\
\hline Blotch & 7.18 & 1.27 & 3.24 & 1.38 & 3.08 & .91 & 3.44 & 1.58 \\
\hline Blouse & 4.57 & 1.51 & 4.40 & 1.11 & 4.21 & .89 & 5.64 & 2.30 \\
\hline Blower & 6.07 & 1.64 & 2.72 & 1.66 & 3.35 & .93 & 4.35 & 1.55 \\
\hline Bodice & 8.22 & .92 & 3.07 & 1.65 & 2.63 & .99 & 3.48 & 2.68 \\
\hline Botany & 8.11 & 1.12 & 2.52 & 1.24 & 3.27 & .96 & 5.31 & 1.89 \\
\hline Bother & 5.00 & 1.50 & 2.30 & 1.45 & 4.45 & .78 & 4.30 & 1.89 \\
\hline
\end{tabular}




\begin{tabular}{|c|c|c|c|c|c|c|c|c|c|c|c|c|c|c|c|c|c|}
\hline \multirow[b]{2}{*}{ Word } & \multicolumn{2}{|c|}{$\begin{array}{c}\text { Age of } \\
\text { Acquisition }\end{array}$} & \multicolumn{2}{|c|}{ Imagery } & \multicolumn{2}{|c|}{ Familiarity } & \multicolumn{2}{|c|}{$\begin{array}{c}\text { Mean- } \\
\text { ingfulness }\end{array}$} & \multirow[b]{2}{*}{ Word } & $\begin{array}{l}\text { Age } \\
\text { Acqui }\end{array}$ & $\begin{array}{l}\text { of } \\
\text { sition }\end{array}$ & Ima & $\mathrm{y}$ & Famil & rity & $\mathrm{Me}$ & n- \\
\hline & Mean & SD & Mean & SD & Mean & $\mathrm{SD}$ & Iean & SD & & Mean & SD & Mean & SD & Mean & $\mathrm{SD}$ & Mean & SD \\
\hline Bounce & 3.84 & 1.78 & 3.26 & 1.37 & 3.78 & .87 & 4.55 & 2.21 & Cobalt & 13 & 1.12 & 3.00 & 1.59 & 3.00 & .88 & 4.28 & 2.05 \\
\hline Bowery & 8.27 & 1.25 & 2.08 & 1.55 & 2.24 & .77 & 2.08 & 2.38 & Cobra & 6.38 & .87 & 5.00 & 1.34 & 4.00 & .90 & 5.35 & 1.87 \\
\hline Bowman & 7.87 & 1.43 & 2.75 & 1.74 & 2.18 & .75 & 3.60 & 2.43 & Combat & 5.94 & 1.30 & 3.73 & 1.40 & 3.56 & .86 & 5.46 & 2.10 \\
\hline Branch & 4.33 & 1.60 & 4.38 & 1.02 & 3.84 & .87 & 4.58 & 2.04 & Comedy & 5.99 & 1.24 & 2.93 & 1.48 & 4.21 & .66 & 5.31 & 1.69 \\
\hline Brandy & 7.23 & 1.30 & 4.24 & 1.24 & 3.80 & .87 & & 1.88 & Consul & 7.94 & 1.19 & 2.47 & 1.45 & 2.76 & .93 & 2.85 & 1.69 \\
\hline Brawl & 7.08 & 1.14 & 3.64 & 1.12 & 2.87 & .99 & & 1.83 & Cornet & & 1.31 & & 1.81 & 2.29 & .80 & & 2.82 \\
\hline Breach & 8.24 & .86 & 1.94 & 1.31 & & .90 & & 2.00 & se & & 1.21 & & .87 & & .82 & & 1.77 \\
\hline Breast & 5.63 & 1.60 & 4.47 & 1.19 & 4 & .73 & & 1.78 & Cor & & & & 1.24 & & .73 & & 1.68 \\
\hline Breath & 4.68 & 1.51 & 2.72 & 1.48 & 4.42 & .68 & & 1.58 & Cor & & .85 & & 1.27 & 8 & .88 & & 2.27 \\
\hline Bridge & 4.67 & 1.57 & 4.59 & .86 & 4.20 & .68 & & 2.95 & h & & 1.25 & & 1.42 & 0 & .54 & & 1.68 \\
\hline Bridle & 5.76 & 1.53 & 4.1 & 1.23 & 3.36 & .92 & 4.8 & 1.45 & ole & & & & & & .59 & & 1.42 \\
\hline Broach & 7.61 & 1.29 & 3.7 & 1.53 & 2.60 & .67 & 3.66 & 1.81 & & & & & & & .45 & & 1.89 \\
\hline Bronze & 6.76 & 1.28 & 3.5 & 1.34 & 3.12 & .78 & 4.7 & 1. & $n$ & & & & & & .82 & & 1.67 \\
\hline Bruise & 4.61 & 1.48 & 4.4 & .88 & 3.89 & .84 & 6.4 & 2. & & & & & & & .76 & & 02 \\
\hline Bucket & 4.22 & 1.33 & 4.4 & 1.08 & 4.00 & .83 & 5. & 1. & & & & & & & .76 & & 62 \\
\hline Buckle & 4.58 & 1.48 & 4.3 & 1.16 & 3. & .74 & & 1. & & & & & & & .75 & & 62 \\
\hline Budget & 7.30 & .99 & 2.7 & 1.4 & & .74 & & 1.2 & Cray oil & & 1.05 & 4.51 & & & .87 & & 57 \\
\hline Bumper & 5.06 & 1.39 & 4.31 & 1.17 & 3.96 & .83 & 5.29 & 1.9 & Credit & 7.05 & 1.25 & 2.40 & 1.46 & 4.14 & .67 & 4.65 & 1.83 \\
\hline Bundle & 5.46 & 1.28 & 4.01 & 1.24 & 3.73 & .72 & 5.04 & 1.9 & 2 & 6.76 & 1.14 & 3.49 & 1.32 & 3.23 & .73 & 5.85 & 1.51 \\
\hline Bunker & 7.33 & 1.39 & 3.38 & 1.57 & 3.07 & 1.05 & 3.67 & 1.9 & $\mathrm{Cu}$ & 8.50 & 1.16 & 1.63 & 1.18 & 1.79 & .96 & 1.48 & 2.11 \\
\hline Burden & 6.82 & 1.32 & 2.53 & 1.31 & 3.58 & 1.04 & 4.48 & 1.5 & Curfew & 7.67 & 1.08 & 2.53 & 1.53 & 3.47 & .90 & 5.32 & 1.89 \\
\hline Burlap & 6.60 & 1.41 & 4.50 & 1.09 & 3.43 & .92 & 5.53 & 1.4 & Cymbal & 6.57 & 1.59 & 3.96 & 1.43 & 3.06 & 1.07 & & 2.34 \\
\hline Butler & 5.81 & 1.15 & 4.27 & 1.25 & 3.21 & .68 & 4.75 & 1.55 & Dan & 7.07 & 1.44 & 2.34 & 1.44 & 3.00 & .85 & 3.43 & 1.99 \\
\hline Bygone & 7.48 & 1.14 & 1.47 & .87 & 2.76 & .81 & 4.07 & 1.80 & Damsel & 6.64 & 1.55 & 4.03 & 1.23 & 2.37 & .58 & 5.44 & 1.94 \\
\hline Caliph & 8.84 & .76 & 1.59 & 1.26 & 1.46 & .58 & 2.24 & 2.89 & Dan & 4.35 & 1.52 & 2.80 & 1.57 & 4.08 & .74 & 5.16 & 3.29 \\
\hline Camper & 5.89 & 1.39 & 3.91 & 1.35 & 3.75 & .82 & 5.99 & 1.75 & $\mathrm{De}$ & 5.76 & 1.53 & 3.24 & 1.56 & 3.13 & .83 & 4.52 & 2.02 \\
\hline Campus & 7.24 & 1.22 & 4.43 & .93 & 4.73 & .57 & 5.38 & 2.28 & Debtor & 7.25 & 1.38 & 2.46 & 1.37 & 2.91 & .74 & 3.78 & 1.69 \\
\hline Candle & 4.29 & 1.23 & 4.79 & .61 & 4.13 & .74 & 6.46 & 2.47 & Denial & 7.19 & 1.47 & 1.86 & 1.31 & 3.38 & .84 & 3.46 & 2.00 \\
\hline Candor & 8.45 & 1.19 & 1.61 & 1.23 & 2.46 & .92 & 1.88 & 2.11 & Design & 6.21 & 1.34 & 3.21 & 1.47 & 3.89 & .80 & 5.48 & 1.78 \\
\hline Canker & 8.10 & 1.45 & 2.19 & 1.54 & 2.30 & .99 & 2.75 & 2.35 & Detail & 6.47 & 1.11 & 1.87 & 1.05 & 4.31 & .73 & 4.72 & 1.49 \\
\hline Canopy & 6.80 & 1.41 & 4.06 & 1.30 & 3.26 & .72 & 6.68 & 8.45 & Dia & 3.59 & 1.48 & 4.65 & .84 & 3.50 & .90 & 5.95 & 2.07 \\
\hline Canter & 7.85 & 1.48 & 2.72 & 1.53 & 2.42 & .91 & 3.92 & 2.15 & Dictum & 8.72 & .70 & 1.25 & .62 & 1.94 & .64 & 2.27 & 1.43 \\
\hline Canyon & 5.91 & 1.29 & 4.74 & .58 & 3.19 & .90 & 5.48 & 1.72 & Dimple & 5.50 & 1.21 & 4.50 & 1.06 & 3.66 & .79 & 4.60 & 1.50 \\
\hline Captor & 7.52 & 1.41 & 2.25 & 1.36 & 2.57 & .93 & 3.15 & 2.48 & Doings & 5.90 & 1.48 & 1.51 & .99 & 3.80 & .98 & 5.59 & 1.90 \\
\hline Carbon & 7.43 & 1.27 & 3.49 & 1.55 & 3.55 & .76 & 6.48 & 2.37 & Domain & 7.57 & 1.10 & $2.60)$ & 1.36 & 3.07 & .65 & 4.75 & 2.11 \\
\hline Carpet & 5.04 & 1.58 & 4.34 & 1.22 & 4.48 & .67 & 6.15 & 1.97 & Donkey & 4.19 & 1.59 & 4.62 & .88 & 3.13 & .86 & 5.67 & 2.07 \\
\hline Carton & 4.59 & 1.40 & 4.22 & 1.00 & 4.13 & .66 & 4.64 & 2.22 & Double & 5.15 & 1.60 & 2.29 & 1.42 & 4.41 & .74 & 4.83 & 1.46 \\
\hline Casing & 7.84 & 1.02 & 2.50 & 1.34 & 2.94 & .87 & 3.23 & 1.45 & Dragon & 4.06 & 1.55 & 4.40 & 1.10 & 3.07 & .90 & 5.86 & 1.77 \\
\hline Casino & 7.67 & 1.08 & 4.20 & 1.12 & 2.75 & .78 & 6.04 & 2.17 & Drink & 1.69 & .99 & 4.43 & 1.11 & 4.73 & .30 & 5.14 & 1.86 \\
\hline Casket & 6.11 & 1.27 & 4.71 & .81 & 3.46 & .83 & 6.64 & 2.42 & & 5.84 & 1.58 & 4.01 & 1.17 & 3.27 & .85 & 5.07 & 1.94 \\
\hline Castle & 4.92 & 1.55 & 4.53 & 1.04 & 3.43 & .89 & 6.53 & 2.03 & End & 8.54 & .67 & 1.21 & 1.29 & 2.87 & .80 & 2.65 & 1.73 \\
\hline Castor & 8.05 & 1.35 & 2.24 & 1.48 & 2.18 & .90 & 2.48 & 1.85 & Fabric & 6.11 & 1.43 & 4.19 & 1.15 & 3.96 & .79 & 6.18 & 2.32 \\
\hline Catnip & 697 & 1.65 & 2.98 & 1.59 & 9 & .74 & 3.56 & 2.10 & Factor & 6.91 & 1.24 & 1.68 & 1.11 & 3.94 & .81 & 4.15 & 1.26 \\
\hline Censor & 7.40 & 1.27 & 2.32 & 1.37 & 3.64 & .72 & 4.45 & 1.94 & on & 6.91 & 1.28 & 4.14 & 1.29 & 2.73 & .97 & 4.92 & 1.55 \\
\hline Chair & 1 & 1.21 & 4.93 & 1.44 & 4.40 & .40 & 5.42 & 1.59 & ly & 3.85 & 1.41 & 4.22 & 1.21 & 4.69 & .50 & 7.19 & 2.56 \\
\hline Chaise & 7.22 & 1.88 & 2.37 & 1.57 & 2.6 & .84 & 2.43 & 2.0 & ac & 7.39 & 1.11 & 2.95 & 1.48 & 3.25 & .79 & 4.82 & 1.81 \\
\hline Change & 4.70 & 1.48 & 2.57 & 1.45 & 4.6 & .6 & & 2.4 & ous & 5.41 & 1.11 & 2.36 & 1.42 & 4.05 & .74 & 5.00 & 1.80 \\
\hline Chapel & 5.48 & 1.32 & 4.63 & .85 & 3. & .78 & & 1.9 & er & 3.04 & 1.62 & 4.74 & .56 & 4.70 & .57 & 5.84 & 2.17 \\
\hline Charge & 5.77 & 1.38 & 2.58 & 1.44 & 4.14 & .69 & & 1. & et & 5.13 & 1.72 & 4.37 & 1.08 & 4.09 & .91 & 5.85 & 2.40 \\
\hline Cherub & 7.94 & 1.42 & 3.13 & 1.58 & 2.40 & .74 & & 2. & & 4.92 & 1.36 & 2.36 & 1.52 & 4.07 & .67 & 4.42 & 1.17 \\
\hline Chint $z$ & 8.48 & 1.01 & 1.68 & 1.11 & 1.86 & .87 & & 2. & & 8.74 & .76 & 1.38 & .83 & 1.60 & .77 & .35 & .95 \\
\hline Chisel & 6.48 & 1.52 & 4.47 & .93 & 3.05 & .88 & & 1. & & 7.83 & 1.11 & 2.40 & 1.54 & 3.48 & .76 & 5.23 & 2.25 \\
\hline Chorus & 5.83 & 1.30 & 4.15 & 1.25 & 3.58 & .6 & 5.64 & 2. & & 8.67 & .89 & 2.20 & 1.44 & 2.83 & .84 & 2.38 & 1.69 \\
\hline Cider & 5.85 & 1.38 & 4.36 & 1.21 & 2.80 & .95 & 5.35 & 1. & & 5.54 & 1.41 & 3.75 & 1.41 & 4.34 & .69 & 5.84 & 1.89 \\
\hline Cinder & 6.57 & 1.59 & 3.73 & 1.48 & 2.81 & .75 & & 1.7 & $\mathrm{Fi}$ & 6.80 & 1.33 & 3.66 & 1.38 & 3.61 & .88 & 4.77 & 1.27 \\
\hline Cipher & 7.92 & 1.21 & 2.36 & 1.51 & 2.69 & .71 & 3.23 & 1.86 & $\mathrm{Fi}$ & 7.05 & 1.27 & 2.98 & 1.45 & 3.31 & 1.00 & 4.56 & 1.56 \\
\hline Clause & 7.69 & 1.17 & 1.75 & 1.27 & 3.51 & .81 & 4.07 & 1.4 & Fin & 7.47 & 1.66 & 2.88 & 1.40 & 2.65 & .87 & 3.43 & 2.33 \\
\hline Clergy & 6.43 & 1.40 & 3.73 & 1.22 & 3.48 & .68 & 5.89 & 2.47 & Fin & 2.87 & 1.29 & 4.79 & .66 & 4.65 & .59 & 6.00 & 2.23 \\
\hline Clerk & 5.85 & 1.28 & 4.00 & 1.33 & 3.60 & .87 & 4.92 & 1.74 & $\mathrm{Fla}$ & 8.65 & .92 & 1.86 & 1.33 & 1.78 & .78 & 1.71 & 2.49 \\
\hline Client & 7.05 & 1.19 & 3.32 & 1.46 & 3.58 & .75 & 4.72 & 2.01 & Fla & 8.53 & 1.05 & 2.15 & 1.49 & 1.93 & .96 & 1.89 & 2.29 \\
\hline Climax & 7.17 & 1.17 & 2.73 & 1.68 & 3.85 & .79 & 4.17 & 1.6 & $\mathrm{Fla}$ & 4.84 & 1.32 & 2.85 & 1.59 & 4.35 & .74 & 6.00 & 2.21 \\
\hline Climb & 2.46 & 1.19 & 3.64 & 1.34 & 3.53 & .74 & 5.00 & 2.0 & & 5.65 & 1.09 & 3.46 & & 3.82 & .84 & & \\
\hline Clique & 8.09 & .95 & 2.14 & 1.47 & 2.96 & .94 & 3.88 & 2.14 & & 7.00 & 1.48 & 2.21 & 1.37 & 2.87 & .87 & 3.57 & 1.68 \\
\hline Closet & 4.12 & 1.58 & 4.46 & .95 & 4.55 & .75 & 5.42 & 2.10 & & 6.38 & 1.61 & 2.64 & 1.19 & 3.33 & .88 & 3.42 & 2.01 \\
\hline Cloth & 4.23 & 1.25 & 4.57 & 1.37 & 4.07 & .88 & 5.00 & 2.15 & & 3.85 & 1.46 & 4.71 & 1.49 & 3.87 & .76 & 5.57 & 1.91 \\
\hline Coarse & 6.51 & 1.25 & 3.25 & 1.30 & 3.72 & .87 & 4.56 & 1.45 & Flower & 3.08 & 1.02 & 4.73 & .75 & 4.36 & .71 & 6.26 & 1.93 \\
\hline
\end{tabular}




\begin{tabular}{|c|c|c|c|c|c|c|c|c|c|c|c|c|c|c|c|c|c|}
\hline \multirow[b]{2}{*}{ Word } & \multicolumn{2}{|c|}{$\begin{array}{c}\text { Age of } \\
\text { Acquisition }\end{array}$} & \multicolumn{2}{|c|}{ Imagery } & \multicolumn{2}{|c|}{ Familiarity } & \multicolumn{2}{|c|}{$\begin{array}{c}\text { Mean- } \\
\text { ingfulness }\end{array}$} & \multirow[b]{2}{*}{ Word } & $\begin{array}{r}\text { Age } \\
\text { Acqui }\end{array}$ & $\begin{array}{l}\text { of } \\
\text { sition }\end{array}$ & $\operatorname{Im}$ & & ar & arity & & \\
\hline & Mean & SD & Mean & SD & Mean & SD & Mean & $\mathrm{SD}$ & & lean & D & Mean & SD & Mean & SD & Tean & $\mathrm{SD}$ \\
\hline ible & & .02 & 1.02 & 1.22 & 1.07 & .88 & .50 & 1.01 & $\mathrm{udo}_{\mathrm{s}}$ & .31 & 1.17 & .57 & 1.31 & 2.53 & .62 & 5.71 & 2.02 \\
\hline Forest & 35 & 1.37 & 4.64 & .94 & 3.94 & .69 & 6.60 & 2.20 & Jumper & 5.43 & 1.77 & & 1.39 & 58 & .84 & 12 & .90 \\
\hline Formal & 6.91 & 1.25 & 3.72 & 1.45 & 3.77 & .84 & 5.32 & 1.89 & Jungle & & 1.18 & 4.36 & 1.13 & & .76 & .23 & 1.66 \\
\hline Fowler & 8.75 & .60 & 1.74 & 1.29 & 2.13 & .76 & 1.81 & 1.77 & Junket & & 1.11 & & 1.58 & & .66 & .20 & .83 \\
\hline Fresco & 8.33 & 1.19 & 2.64 & 1.63 & 2.46 & .97 & 3.08 & 3.00 & & & 1.04 & & & & .73 & 57 & 2.60 \\
\hline Friend & 3.36 & 1.18 & 4.09 & 1.06 & 4.81 & .44 & 5.35 & 1.52 & & & 1.30 & & 1.50 & & .88 & .56 & 1.58 \\
\hline Fright & 4.80 & 1.60 & & 1.48 & 3.95 & .93 & 5.12 & 2.07 & & & 1.41 & & .87 & & .84 & .65 & 2.26 \\
\hline Fringe & 6.15 & 1.64 & & 1.29 & & .76 & 4.26 & 1.23 & & & 1.46 & & 1.01 & & .78 & 98 & 2.10 \\
\hline & 3.31 & 1.23 & 4.79 & 1.22 & & .56 & 6.28 & 1.56 & & & 1.12 & & 1.45 & & .90 & 42 & 1.63 \\
\hline nble & 6.27 & 1.37 & 3.67 & 1.42 & & .82 & 4.16 & 1.46 & & & .94 & & & & .94 & 18 & 2.59 \\
\hline ion & 7.98 & 1.21 & 2. & 1.46 & & .84 & 4.04 & 1.35 & & & 1.18 & & 1.28 & & .74 & 34 & 2.84 \\
\hline ty & 7.33 & 1.27 & 2.66 & 1.37 & & .86 & 4.65 & 1.67 & & & 1.79 & 4.07 & 1.41 & 3.57 & .93 & .30 & 2.35 \\
\hline der & 7.18 & 1.52 & 2.9 & 1.57 & & .97 & 4.19 & 2.69 & & & 1.29 & 3.79 & 1.28 & 3.32 & .85 & 5.08 & 2.25 \\
\hline len & 4.10 & 1.59 & 4.65 & .68 & 4. & .91 & 7.36 & 2.14 & & & 1.10 & 1.60 & 1.14 & 2.22 & .79 & 2.77 & 2.05 \\
\hline lic & 6.14 & 1.47 & 4.37 & $1: 11$ & 3. & .72 & 6.21 & 1.76 & ut & & 1.23 & 2.66 & 1.41 & 3.41 & .85 & 10 & 1.38 \\
\hline net & 7.67 & 1.52 & 2.81 & 1.56 & 2.5 & .78 & 4.25 & 2.10 & $y$ & 1 & .84 & 2.11 & 1.16 & 3. & .83 & 8 & 1.87 \\
\hline & 6.70 & 1.16 & 2.70 & 1.48 & 3.91 & .82 & 4.23 & 1.14 & in & & 1.23 & 2.65 & 1.50 & 2. & .75 & 8 & 1.64 \\
\hline & 8.10 & 1.22 & 1.86 & 1.22 & 2.63 & .73 & 2.75 & 1.79 & th & 0 & 1.21 & 2.54 & 1.40 & 4.20 & .70 & 9 & .79 \\
\hline oul & 7.69 & 1.54 & 3.14 & 1.46 & 2.00 & .98 & 4.21 & 2.04 & & 8.51 & .97 & 1.91 & 1.16 & & .89 & 3 & .92 \\
\hline Giant & 2.77 & 1.09 & 4.93 & 1.33 & $3.40^{\circ}$ & .92 & 4.28 & 1.95 & hen & 7.98 & 1.42 & 2.75 & 1.65 & 2. & .82 & 5 & 3.13 \\
\hline le & 5.91 & 1.51 & 4.15 & 1.41 & 3.19 & .92 & 4.83 & 1.95 & & 8.16 & 1.45 & 1.79 & 1.37 & 9 & .98 & 2.49 & 2.63 \\
\hline ice & 6.32 & 1.31 & 2.80 & 1.49 & 4.21 & .87 & 5.05 & 1.68 & & 7.71 & 1.38 & 2.43 & 1.58 & 2. & .69 & 3.96 & 2.30 \\
\hline ler & 6.47 & 1.41 & 4.24 & 1.21 & 3.2 & .90 & 5.22 & 1.83 & & 5.19 & 1.60 & 4.68 & .88 & 3. & .91 & 5. & 1.73 \\
\hline Gloat & 8.00 & 1.11 & 1.71 & 1.51 & 2.8 & .94 & 3.07 & 1.99 & & 2 & 1.29 & 3.03 & 1.42 & 3. & .70 & 4. & 1.38 \\
\hline ten & 8.01 & 1.41 & 2.49 & 1.47 & 2.72 & 1.07 & 4.35 & 2.84 & & & 1.37 & 3.98 & 1.23 & 3. & .96 & 6 & 2.26 \\
\hline Goblet & 6.42 & 1.49 & 4.41 & 1.14 & 3.01 & .90 & 5.59 & 2.38 & & & 1.46 & 4.41 & 1.21 & 8 & .87 & 0 & 1.41 \\
\hline Gopher & 5.60 & 1.42 & 4.23 & 1.17 & 2.8 & .77 & 4. & 2.07 & & 4 & 1.41 & 4.57 & .81 & 3 & .90 & 0 & 1.73 \\
\hline Gospel & 6.04 & 1.47 & 2.95 & 1.49 & 3. & .75 & 5.92 & 1.87 & & & 1.54 & 3. & 1.35 & & .77 & & 2.04 \\
\hline Gravel & 4.71 & 1.44 & 4.35 & 1.02 & 3. & .87 & 5. & 1.53 & & 6 & 1.42 & 0 & 1.52 & 2 & .84 & 2 & 2.25 \\
\hline Groin & 8.08 & 1.00 & 4.07 & 1.35 & 2.67 & 1.05 & 2.92 & 1.62 & & & 1.11 & 0 & 1.39 & & .78 & 1 & 1.88 \\
\hline Grouch & 5.53 & 1.69 & 3.22 & 1.53 & 3.95 & .92 & 4.43 & 2.01 & & 7 & 1.28 & 3 & 1.22 & 2 & .87 & & 2.52 \\
\hline Ground & 3.76 & 1.51 & 4.38 & 1.22 & 4.4 & .83 & 6.36 & 1.73 & $y$ & 6 & 1.16 & 2.34 & 1.50 & 2. & .91 & 8 & 1.56 \\
\hline Grouse & 8.09 & 1.38 & 2.71 & 1.73 & 2.3 & .87 & 3.88 & 2.42 & et & 8 & 1.32 & 4.44 & 1.04 & 3. & .82 & 2 & 1.84 \\
\hline Growth & 5.19 & 1.45 & 2.60 & 1.42 & 4.17 & .84 & 4.92 & 1.88 & & 7.17 & 1.71 & 3.26 & 1.63 & 2.41 & .69 & 2.77 & 2.32 \\
\hline Guard & 5.23 & 1.14 & 4.00 & 1.13 & 3.3 & .85 & 4.78 & 1.06 & & 7.98 & 1.14 & 1.84 & 1.03 & 3.11 & .90 & 3.84 & 1.82 \\
\hline Guide & 5.46 & 1.28 & 2.93 & 1.37 & 3.8 & .83 & 4.21 & 1.58 & & 4. & 2.09 & 3.88 & 1.47 & 8 & .81 & 7 & 1.94 \\
\hline & 5.07 & 1.45 & 4.66 & .81 & 4.0 & .91 & 6.36 & 2.09 & & 0 & 1.82 & 4.04 & 1.51 & & .88 & 9 & 1.65 \\
\hline ter & 7.05 & 1.36 & 3.36 & 1.52 & 2.6 & .82 & 4.16 & 1.86 & & & 1.72 & 8 & 8 & 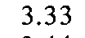 & .84 & 4 & 1.45 \\
\hline it & 5.53 & 1.38 & 1.50 & 1.45 & 4. & .88 & 3.64 & 2.23 & & 6 & 1.66 & 4. & 1.05 & 3. & .81 & 6. & 2.05 \\
\hline er & 6.60 & 1.61 & 4. & 1.26 & 3 & .89 & 4.08 & 1.77 & & 5. & 1.38 & 3. & 1.41 & 4.0 & .76 & 4.55 & 1.48 \\
\hline le & 4.86 & 1.40 & 3. & 1.42 & 4 & .74 & 4. & 1.85 & & 5.08 & 1.37 & 4.22 & 1.16 & 3.8 & .90 & 5.77 & 2.34 \\
\hline & 4.62 & 1.38 & 4 & 1.18 & 4 & .91 & & 2.23 & arvel & 6.79 & 1.16 & 2.03 & 1.29 & 3.2 & .75 & 4.24 & 1.76 \\
\hline & 8.2 & 1.61 & & 1.5 & 2 & 1.00 & 3 & 2.21 & & 7.58 & 1.80 & 2.48 & 1.52 & 2.43 & .81 & 3.03 & 2.21 \\
\hline & 5.3 & 1.39 & & 1. & & .86 & 4 & 1.49 & & 7.97 & 1.08 & 3.09 & 1.42 & 2.66 & .69 & 3.75 & 1.90 \\
\hline$c$ & 8 & .97 & & 1.32 & & .94 & 3.14 & 1.61 & & 7.68 & 1.24 & 3.10 & 1.51 & 3.58 & .88 & 4.27 & 1.28 \\
\hline or & 8.54 & .91 & 1.92 & 1.48 & 2.3 & .70 & 1.76 & 1.79 & ody & 6.04 & 1.25 & 2.80 & 1.60 & 4.05 & .77 & 5.46 & 1.93 \\
\hline ald & 7.20 & 1.54 & 2.41 & 1.43 & 3.9 & .94 & 3.65 & 1.29 & lentor & 8.33 & 1.23 & 1.91 & 1.10 & 2.33 & .91 & 1.48 & 1.81 \\
\hline mit & 6.61 & 1.26 & 4.17 & 1.22 & 3.1 & .87 & 5.33 & 2.51 & ino & 8.73 & .83 & 1.57 & 1.13 & 1.43 & .59 & 1.08 & 1.65 \\
\hline & 6.50 & 1.28 & 4.3 & 1.13 & 3.5 & .8 & 6.5 & 2.24 & od & 6.45 & 1.12 & 1.92 & 1.30 & 3.98 & .77 & 4.12 & 1.72 \\
\hline ge & 8.29 & .96 & & 1.17 & 2.7 & .80 & 3.0 & 2.0 & & 5.66 & 1.37 & 4.49 & 1.04 & 2.92 & .94 & 4.74 & 1.75 \\
\hline & 5.36 & 1.61 & 4.2 & 1.20 & 3.1 & .72 & 5.72 & 1.9 & & 6.06 & 1.27 & 2.77 & 1.48 & 3.65 & .92 & 4 & 1.74 \\
\hline & 2.31 & 1.12 & 4.9 & 1.3 & 4.5 & .45 & 5.5 & 2.0 & & 6.77 & 1.37 & 3.65 & 1.33 & 3. & .79 & 5.11 & 1.67 \\
\hline & 6.84 & 1.56 & 2.1 & 1.3 & 2.3 & .7 & 3.5 & 1.8 & & 4.39 & 1.37 & 2.60 & 1.49 & 4.8 & .49 & 4 & 1.86 \\
\hline & 4.31 & 1.23 & 4.9 & 1.19 & 4.0 & .86 & 5.28 & 1.6 & & 7.27 & 1.09 & 3.06 & 1.40 & 2. & .85 & 4 & 2.14 \\
\hline & 7.04 & 1.29 & $4 .($ & 1.14 & 3.1 & .7 & 5.12 & 1.6 & & 6.42 & 1.32 & 2.75 & 1.43 & 3.81 & .85 & 4 & 1.47 \\
\hline & 7.19 & 1.27 & 3. & 1.3 & 2.9 & .83 & 3.84 & 2.0 & & 7.12 & 1.29 & 2.68 & 1.31 & 3.47 & .78 & 3.91 & 1.75 \\
\hline rid & 7.93 & 1.11 & 2. & 1.60 & 3.1 & .90 & 4.50 & 2.4 & & 7.56 & 1.28 & 2.55 & & 2.65 & .81 & 2.59 & 1.53 \\
\hline & 8.38 & .67 & & 1.16 & 2.8 & 1.02 & 2.42 & 1.8 & & 4.85 & 1.26 & 4.21 & & & .84 & & 1.50 \\
\hline & 3.66 & 1.31 & 4.52 & .97 & 4.4 & .65 & 5.92 & 2.34 & lonkey & 4.07 & 1.49 & 4.46 & 1.11 & & .76 & 5.71 & 1.88 \\
\hline Jailor & 6.16 & 1.27 & 4.25 & 1.15 & 3.11 & .80 & 4.18 & 1.66 & Ionth & 3.92 & 1.05 & 2.57 & 1.22 & 4.27 & .79 & 4.64 & 1.51 \\
\hline Jargon & 8.12 & 1.06 & 1.82 & 1.08 & 3.01 & .81 & 3.26 & 1.79 & Iorale & 7.61 & 1.09 & 2.16 & 1.46 & 3.87 & .72 & 4.31 & 2.19 \\
\hline Jasper & 7.57 & 1.78 & 1.84 & 1.24 & 2.31 & .76 & 2.44 & 1.83 & forgue & 7.34 & 1.35 & 3.59 & 1.43 & 2.93 & .82 & 4.53 & 1.78 \\
\hline Jaunt & 8.54 & 1.82 & 2.07 & 1.15 & 2.47 & .91 & 2.42 & 1.62 & Morsel & 6.90 & 1.28 & 3.22 & 1.28 & 3.35 & .87 & 4.68 & 2.12 \\
\hline & 8.43 & 1.11 & 1.66 & 1.16 & 1.92 & .84 & 1.90 & 1.86 & Mortal & 7.04 & 1.27 & 2.76 & 1.62 & 3.36 & .75 & 4.56 & 1.78 \\
\hline & 6.45 & 1.24 & 4.39 & 1.12 & 3.47 & .71 & 6.18 & 2.24 & Mosaic & 7.71 & 1.16 & 3.74 & 1.48 & 3.23 & .78 & 4.50 & 2.10 \\
\hline Joust & 7.77 & 1.02 & 3.35 & 1.45 & 2.33 & .85 & 4.78 & 1.44 & Mosque & 8.33 & .95 & 2.77 & 1.66 & 2.33 & .72 & 2.81 & 2.76 \\
\hline
\end{tabular}




\begin{tabular}{|c|c|c|c|c|c|c|c|c|c|c|c|c|c|c|c|c|c|}
\hline \multirow[b]{2}{*}{ Word } & \multicolumn{2}{|c|}{$\begin{array}{c}\text { Age of } \\
\text { Acquisition }\end{array}$} & \multicolumn{2}{|c|}{ Imagery } & \multicolumn{2}{|c|}{ Familiarity } & \multicolumn{2}{|c|}{$\begin{array}{c}\text { Mean- } \\
\text { ingfulness }\end{array}$} & \multirow[b]{2}{*}{ Word } & $\begin{array}{l}\text { Age } \\
\text { Acquis }\end{array}$ & $\begin{array}{l}\text { of } \\
\text { sition }\end{array}$ & & & 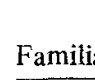 & & & ess \\
\hline & Mean & SD & Mean & $\mathrm{SD}$ & Mean & SD & Mean & SD & & n & D & Mean & D & n & D & ean & D \\
\hline Mother & 3.00 & .47 & 76 & .55 & 84 & .36 & 55 & 56 & lish & 07 & 58 & 1. & 1.47 & 5 & .87 & 5.69 & .82 \\
\hline & & .06 & & 1.20 & & .78 & & & & & 1.19 & & & & .80 & & 2.32 \\
\hline Muscle & 5.56 & 1.55 & & 1.05 & & .75 & & & & & .75 & & & & .73 & & 2.28 \\
\hline Music & 3.08 & 1.29 & & 1.20 & 4.60 & .48 & & & ound & 4.77 & 1.15 & & & & .77 & & 1.39 \\
\hline Musket & 6.53 & 1.39 & & 1.47 & & .81 & & & uter & 7.41 & 2.02 & 2.04 & 1.46 & & .85 & & 2.30 \\
\hline Muslin & 7.71 & 1.30 & .29 & 1.55 & 05 & .84 & 3.60 & & Powder & 5.09 & 1.87 & 4.17 & 1.04 & & .78 & & 1.98 \\
\hline Mutiny & 7.37 & 1.16 & 270 & 1.50 & & .57 & & 1.66 & Power & 5.23 & 1.17 & 1.71 & 1.36 & & .74 & 4.78 & 1.54 \\
\hline Myrtle & 7.82 & 1.53 & 2.24 & 1.59 & 48 & .85 & 2.83 & 1.34 & Praise & 5.73 & 1.50 & 2.35 & 1.46 & & .72 & 3.89 & 2.00 \\
\hline Mystic & 7.72 & 1.21 & 2.56 & 1.51 & 3.17 & .78 & 4.92 & 1.91 & & 6.42 & 1.10 & 2.59 & 1.64 & 3.51 & 1.01 & 4.28 & 1.90 \\
\hline Napkin & 4.22 & 1.43 & 4.63 & .86 & 4.54 & .67 & 5.92 & 1.87 & & 4.06 & 1.30 & 4.10 & 1.37 & & .82 & & 1.57 \\
\hline Native & 6.35 & 1.14 & 3.74 & 1.23 & 3.66 & .90 & 4.84 & 1.57 & & & 1.25 & & .97 & & .74 & & 1.39 \\
\hline Nature & 5.31 & 1.46 & 3.86 & 1.46 & 34 & .56 & 6.03 & 2.03 & & 6.79 & 1.22 & & 1.57 & & .70 & & 1.57 \\
\hline Nebula & 8.77 & .53 & .83 & 1.39 & .21 & .84 & 3.15 & 2.95 & & 6.21 & & & & & .74 & & 1.70 \\
\hline Nectar & 7.07 & 1.42 & 3.19 & 1.45 & 73 & .91 & & 1.56 & & & & & & & .78 & & 2.79 \\
\hline Nicety & 7.25 & 1.52 & 1.60 & .83 & 68 & .81 & 3.90 & 2.98 & & & & & & & .76 & & 73 \\
\hline Nickle & 4.15 & 1.65 & 4.50 & .94 & 4.62 & .74 & & 1.96 & & & 1.39 & & & & .84 & & \\
\hline Noble & 6.77 & 1.16 & 2.07 & 1.37 & 2.93 & 1.00 & 4. & 1.68 & & & 1.05 & & & & .92 & & 1.91 \\
\hline Nodule & 8.15 & 1.63 & 2.65 & 1.56 & 2.48 & .89 & 3. & 2. & & & & & & & .82 & & 1.88 \\
\hline Notary & 8.07 & 1.19 & 1.80 & 1.15 & 3.05 & .77 & 4. & 2. & & & & & 1.37 & & .88 & & 1.83 \\
\hline Notice & 5.91 & 1.37 & 2.9 & 1.44 & 4.28 & .77 & & & & 7.71 & 1.32 & & 1. & & .78 & & 1.52 \\
\hline Novice & 7.82 & 1.25 & 2.2 & 1.41 & & .95 & & & & 7.42 & 1.07 & & & & .88 & & 1.16 \\
\hline Nurt & 3.58 & 1.31 & 3.7 & 1.36 & & .68 & 4.64 & 2.29 & & & & & & & .78 & & 1.84 \\
\hline Nutmeg & 7.01 & 1.33 & 3.5 & 1.4 & 3.05 & .73 & 4.51 & 1.87 & & & & & & & .84 & & 1.99 \\
\hline Nymph & 8.15 & 1.10 & 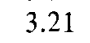 & 1.47 & 2.60 & .94 & 3.9 & 1.8 & & & & & & & .67 & & 1.92 \\
\hline Opi & 8 & .7 & 3.7 & 1.41 & 2.93 & 1.04 & 5.0 & 1.8 & & & & & & & .71 & & 2.24 \\
\hline Packet & 6.32 & 1.28 & 3.6 & 1.20 & 3.43 & .90 & 41 & & & & & & & & .52 & & 1.26 \\
\hline Paint & 4.23 & 1.02 & .43 & 10 & 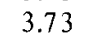 & .75 & 6.0 & 1. & & & 1. & & & & .83 & & 2.23 \\
\hline Panic & 6.69 & .94 & 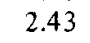 & 13 & 9 & .76 & 4. & 1.8 & & & 1.60 & & & & .78 & & 1.79 \\
\hline Pantry & 6.36 & 1.55 & 7 & 123 & & .76 & 5 & 2. & & & 1.18 & & & & .74 & & 1.39 \\
\hline Parcel & 7.08 & 1.32 & .7 & 13 & & .81 & 5. & 1. & & 8 & $c$ & & & & .80 & & 2.48 \\
\hline Parent & 4.11 & 1.41 & 38 & 1 & & .66 & 56 & 1. & & 3 & 1.06 & & & & .81 & & 1.87 \\
\hline Parish & 6.92 & 1.47 & 8 & 1.53 & 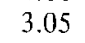 & .88 & 4.11 & 1.8 & & 7 & 1.34 & 2. & 1.42 & & .70 & & 1.87 \\
\hline ody & 8.70 & .67 & 15 & .85 & & .87 & 2 & 2.4 & & & 1.64 & 4 & 1.41 & 3.60 & 1.08 & & 2.25 \\
\hline role & 7.75 & 1.01 & 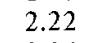 & 1.40 & & .86 & 4.42 & 1.4 & & & 1.33 & & 1. & 3. & .82 & & 1.65 \\
\hline irson & 7.06 & 1.33 & 3.5 & 15 & & .71 & 3. & 1. & & 5 & 1. & & & & .89 & & 1.91 \\
\hline ity & 3.85 & 1.08 & & 11 & 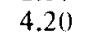 & .63 & 5. & 2. & & 6 & 1. & & & & .82 & & 2.31 \\
\hline astry & 5.71 & 1.66 & 4.4 & 1 & & .76 & 5. & 1. & & 5 & 1. & & 1. & & .81 & & 1.67 \\
\hline thos & 8.90 & .60 & & 1 & & .80 & 1. & 2.2 & & 5 & & & 1.42 & & .63 & 4.81 & 1.66 \\
\hline tio & 5.53 & 1.28 & 4.57 & 1.24 & 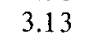 & .95 & 4.50 & 1.7 & & 7.03 & 1.20 & 8 & 1.37 & & .75 & 4.21 & 1.27 \\
\hline rol & 6.08 & 1.12 & 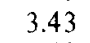 & 1.2 & 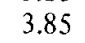 & .79 & 5.11 & 1. & & 2 & 1.12 & 2.11 & 1.43 & & .71 & 3.92 & 1.72 \\
\hline tron & 7.54 & 1.04 & 3 & 1 & & .93 & 3.61 & 1.7 & & & 1. & 4 & .87 & & .81 & & 1.89 \\
\hline Paunch & 8.34 & 1.08 & 1.9 & 1 & 2 & .95 & 1. & 2. & & 2 & & 7 & & & .74 & & 1.30 \\
\hline Pause & 6.15 & 1.08 & 2.21 & 1.3 & 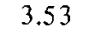 & .98 & 4.4 & 1.8 & & 2 & & 4 & & & .77 & & 2.02 \\
\hline Peanut & 3.39 & 1.14 & 4.44 & 1.15 & 4. & .6 & 5. & 1.6 & & & & 3.44 & & & .84 & & 3.05 \\
\hline Pectin & 8.40 & 1.44 & 1.5 & 1.13 & 1.98 & .78 & 1. & 1. & & & & 5 & & & .57 & & 2.00 \\
\hline Pencil & 3.15 & 1.08 & 4.82 & .60 & 4.76 & .50 & 6. & 2. & & & & 0 & & & .86 & & 1.94 \\
\hline Peony & 7.54 & 1.54 & 3.00 & 1.70 & 2.07 & 1.03 & 3.00 & 2.7 & & 6.31 & 1. & 3. & & & .73 & & 1.73 \\
\hline Period & 5.36 & 1.25 & 3.01 & 1.64 & 4.45 & .64 & 5.29 & 1.8 & & 7.69 & 1.45 & 3. & & & .78 & & 2.41 \\
\hline Permit & 6.24 & 1.37 & 2.86 & 1.55 & 3.80 & .79 & 3.79 & 1.0 & & 8.84 & .49 & 1.7 & 1. & & .61 & & 1.79 \\
\hline Person & 4.35 & 1.78 & 3.86 & 1.37 & 4.75 & .48 & 5.45 & 1.52 & & 5.21 & 1.44 & 2.7 & 1.5 & & .83 & & 2.02 \\
\hline Phrase & 6.34 & 1.23 & 2.19 & 1.42 & 3.90 & .77 & 4.34 & 1.49 & exton & 8.17 & 1.23 & 2.67 & 1.61 & 2 & .70 & 2.46 & 2.34 \\
\hline Physic & 7.76 & 1.30 & 2.10 & 1.30 & 3.46 & 1.01 & 3.96 & 2.1 & haker & 5.73 & 1.68 & 3.56 & 1.36 & 3.58 & .96 & 4.07 & 1.27 \\
\hline Picker & 6.32 & 1.54 & 2.30 & 1.30 & 2.82 & .97 & 4.24 & 2.3 & ty & 7.51 & 1.17 & 3.54 & 1.51 & 2.85 & .81 & 5.32 & 2.15 \\
\hline Picket & 7.14 & 1.39 & 3.44 & 1.30 & 3.47 & .85 & 4.40 & 1.8 & & 6.18 & 1.39 & 4.1 & 1. & 3. & .78 & 4.97 & 1.70 \\
\hline Pickle & 4.40 & 1.56 & 4.69 & .68 & 4.13 & .74 & 5.75 & 2.6 & $\mathrm{Sh}$ & 5.21 & 1.83 & 3.2 & 1. & 3. & .98 & 5.41 & 1.82 \\
\hline Pigeon & 5.31 & 1.40 & 4.73 & .60 & 3.62 & .87 & 5.33 & 1.9 & & 6.90 & 1.2 & 2.8 & 1. & 3.19 & .87 & 5.04 & 1.79 \\
\hline Pirate & 4.92 & 1.29 & 4.69 & .75 & 3.12 & .89 & 5.81 & 1.9 & & 8. & 1. & 1. & 1. & 1.44 & .63 & 1.76 & 2.42 \\
\hline Pistol & 4.94 & 1.42 & 4.62 & .96 & 3.60 & .81 & 5.68 & 1.7 & & 6.60 & 1. & 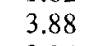 & 1.13 & 3.00 & .84 & 4.92 & 1.60 \\
\hline Plague & 7.15 & 1.36 & 3.11 & 1.40 & 3.24 & .90 & 5.21 & 1.4 & & 7.81 & 1.37 & 3.06 & 1.52 & 2.51 & .80 & 3.78 & 2.48 \\
\hline Planet & 5.82 & 1.28 & 4.21 & 1.11 & 3.64 & .81 & 6.61 & 2.2 & & 7.24 & 1.44 & 3.96 & 1.36 & 2.80 & .72 & 4.26 & 2.21 \\
\hline Plight & 7.52 & 1.19 & 2.31 & 1.35 & 2.91 & .72 & 2.64 & 1.5 & & 5.41 & 1.25 & 3.44 & 1.43 & 4.19 & .84 & 5.73 & 2.24 \\
\hline Pocket & 3.80 & 1.26 & 4.45 & 1.10 & 4.44 & .57 & 5.32 & 1.5 & & 8.36 & 1.18 & 2.40 & 1.53 & 2.26 & .68 & 1.96 & 2.03 \\
\hline Poetry & 5.98 & 1.24 & 3.14 & 1.53 & 3.82 & .71 & 5.37 & 2.2 & & 6.37 & 1.49 & 3.25 & 1.63 & 3.06 & .86 & 4.50 & 2.14 \\
\hline Poker & 5.15 & 1.34 & 4.07 & 1.15 & 3.07 & .93 & 6.42 & 2.1 & & 6.14 & 1.26 & 3.68 & 1.36 & 3.67 & .74 & 5.12 & 1.86 \\
\hline Police & 4.18 & 1.25 & 4.36 & 1.16 & 4.32 & .63 & 6.31 & 2.03 & & 4.21 & 1.39 & 4.61 & .86 & 3.25 & .94 & 5.84 & 2.14 \\
\hline Policy & 7.35 & 1.19 & 2.01 & 1.23 & 3.90 & .90 & 4.54 & 1.39 & Slouch & 6.97 & 1.56 & 3.26 & 1.28 & 3.23 & .92 & 4.16 & 1.99 \\
\hline
\end{tabular}




\begin{tabular}{|c|c|c|c|c|c|c|c|c|c|c|c|c|c|c|c|c|c|}
\hline \multirow[b]{2}{*}{ Word } & \multicolumn{2}{|c|}{$\begin{array}{c}\text { Age of } \\
\text { Acquisition }\end{array}$} & \multicolumn{2}{|c|}{ Imagery } & \multicolumn{2}{|c|}{ Familiarity } & \multicolumn{2}{|c|}{$\begin{array}{c}\text { Mean- } \\
\text { ingfulness }\end{array}$} & \multirow[b]{2}{*}{ Word } & \multicolumn{2}{|c|}{$\begin{array}{c}\text { Age of } \\
\text { Acquisition }\end{array}$} & \multicolumn{2}{|c|}{ Imagery } & \multicolumn{2}{|c|}{ Familiarity } & & \\
\hline & Mean & $\mathrm{SD}$ & Mean & SD & Mean & SD & Mean & SD & & Mean & $\mathrm{SD}$ & Mean & SD & Mean & SD & Mean & SD \\
\hline Sluice & 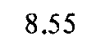 & .97 & 5 & 1.08 & 8 & .95 & 70 & 2.05 & oi & 7 & 1.41 & 2.69 & 1.Ju & 2 & .63 & .69 & 1.89 \\
\hline Smudge & 5.85 & 1.45 & .05 & 1.19 & 3.61 & .78 & 4.92 & 1.66 & Tongue & 3.65 & 1.58 & 4.68 & .92 & so & .66 & .00 & 2.18 \\
\hline Socket & 6.28 & 1.55 & 4.01 & 1.20 & 3.52 & .77 & 4.84 & 1.62 & Tonic & 7.00 & 1.10 & 3.42 & 1.14 & 2.53 & .92 & 4.14 & 1.39 \\
\hline Sodium & 7.94 & .99 & 2.72 & 1.62 & 3.35 & .89 & 4.58 & 1.71 & Tonsil & 5.29 & 1.34 & 3.71 & 1.29 & 3 & .82 & 4.76 & 1.98 \\
\hline Solace & 8.22 & 1.17 & 1.46 & 1.02 & 2.66 & .93 & 2.70 & 2.33 & Train & 3.92 & 1.10 & 5.00 & 1.23 & 7 & .68 & 35 & 1.56 \\
\hline Sphinx & 7 & 1.10 & 4,15 & 1.29 & 2.68 & .74 & 4.69 & 2.07 & Trance & 7.13 & 1.33 & 2.63 & 1.35 & & .90 & 6 & 1.47 \\
\hline Spider & 4 & 1.19 & .52 & 1.14 & 3.83 & .71 & 5.43 & 1.99 & Travel & 5.05 & 1.17 & 3.14 & 1.59 & 3 & .73 & 69 & 1.57 \\
\hline Spigot & 7 & 1.68 & 3.09 & 1.75 & 2.35 & 1.05 & 2.84 & 2.98 & Trench & 6.64 & 1.28 & 4.11 & 1.27 & 5 & .81 & 1 & 1.61 \\
\hline Splint & & 1.24 & 2 & 1.16 & 3.12 & .71 & 4.51 & 1.50 & $\begin{array}{l}\text { Irench } \\
\text { Trend }\end{array}$ & 7.69 & $\begin{array}{r}.20 \\
.96\end{array}$ & 1.21 & 1.37 & 7 & .80 & 4 & 1.68 \\
\hline Sp & & 1.38 & 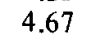 & .90 & 4.08 & .73 & 5.85 & 2.01 & $\begin{array}{l}\text { Irend } \\
\text { Triad }\end{array}$ & $\begin{array}{l}1.09 \\
8.54\end{array}$ & 1.06 & 1.93 & 1.32 & 2.27 & 1.05 & 0 & 1.74 \\
\hline Sprain & & 1.34 & & 1.54 & & .71 & & 1.96 & $\begin{array}{ll}1 \mathrm{ri} \\
\mathrm{Tr} \mathrm{i}\end{array}$ & $\begin{array}{l}0.04 \\
7.97\end{array}$ & $\begin{array}{l}1.00 \\
1.10\end{array}$ & $\begin{array}{l}1.90 \\
1.82\end{array}$ & 1.07 & 5 & .88 & 3.35 & 1.57 \\
\hline Sprout & & 1.32 & & 1.3 & 3 & .72 & & 1.62 & Ir & $\begin{array}{l}1.91 \\
7.60\end{array}$ & $\begin{array}{l}1.10 \\
1.43\end{array}$ & $\begin{array}{l}1.02 \\
3.89\end{array}$ & $\begin{array}{l}1.07 \\
1.43\end{array}$ & 1 & $\begin{array}{l}.00 \\
.78\end{array}$ & 4.19 & 1.67 \\
\hline Spruce & & 1.24 & 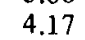 & 1.2 & 3. & .82 & & 1.66 & $\begin{array}{l}\text { Irip } \\
\text { Tro }\end{array}$ & $\begin{array}{l}1.00 \\
5.75\end{array}$ & $\begin{array}{l}1.43 \\
1.25\end{array}$ & $\begin{array}{l}3.09 \\
4.51\end{array}$ & $\begin{array}{l}1.45 \\
1.03\end{array}$ & & .92 & 43 & 1.73 \\
\hline Square & & 1.56 & 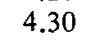 & 1.2 & 3. & .85 & & 2.24 & $\begin{array}{l}\text { Irophy } \\
\text { Trough }\end{array}$ & $\begin{array}{l}5.10 \\
7.04\end{array}$ & $\begin{array}{l}1.20 \\
1.41\end{array}$ & $\begin{array}{l}4.01 \\
3.29\end{array}$ & $\begin{array}{l}1.03 \\
1.67\end{array}$ & & 1.15 & 73 & 2.13 \\
\hline Squawk & 5.90 & 1.80 & 2 & 1.4 & 2. & .84 & & 1.49 & $\begin{array}{l}\text { Trough } \\
\text { Trowel }\end{array}$ & 7.67 & $\begin{array}{l}1.41 \\
1.65\end{array}$ & & $\begin{array}{l}1.07 \\
1.61\end{array}$ & & $\begin{array}{r}1.13 \\
.95\end{array}$ & 44 & $\begin{array}{l}2.13 \\
2.94\end{array}$ \\
\hline Squeak & 4.22 & 1.50 & & 1.6 & 3 & .92 & & 1.93 & $\begin{array}{l}\text { Trowel } \\
\text { Truism }\end{array}$ & 8.54 & $\begin{array}{r}1.03 \\
.75\end{array}$ & $\begin{array}{l}2 . \\
1 .\end{array}$ & $\begin{array}{r}1.01 \\
.93\end{array}$ & & $\begin{array}{l}.95 \\
1.00\end{array}$ & & $\begin{array}{l}2.94 \\
1.49\end{array}$ \\
\hline Squeal & 5.5 & 1.67 & 2. & 1.5 & & .98 & & 2.00 & $\begin{array}{l}\text { Truism } \\
\text { Tubing }\end{array}$ & 6.85 & 1.48 & 3.57 & $\begin{array}{r}.93 \\
1.32\end{array}$ & & $\begin{array}{l}1.00 \\
1.01\end{array}$ & & $\begin{array}{l}1.49 \\
2.51\end{array}$ \\
\hline Squint & 6 & 1.37 & 3.92 & 1.28 & 3 & .75 & & 2.07 & $\begin{array}{l}\text { Tubing } \\
\text { Tucker }\end{array}$ & 7.79 & 1.50 & 1.71 & $\begin{array}{l}1.32 \\
1.26\end{array}$ & & $\begin{array}{r}1.01 \\
.83\end{array}$ & & $\begin{array}{l}2.51 \\
1.89\end{array}$ \\
\hline Squire & 7.44 & 1.23 & 2.81 & 1.46 & 2.7 & .76 & & 2.09 & $\begin{array}{l}\text { ucker } \\
\text { urban }\end{array}$ & 7.22 & 1.16 & 4.01 & $\begin{array}{l}1.20 \\
1.29\end{array}$ & & $\begin{array}{l}.03 \\
.67\end{array}$ & & $\begin{array}{l}1.89 \\
2.10\end{array}$ \\
\hline Squirm & 5.62 & 1.68 & 3 & 1.51 & 3.32 & .85 & & 1.76 & n & 4.09 & 1.51 & 4.84 & $\begin{array}{r}1.29 \\
.40\end{array}$ & & $\begin{array}{l}.07 \\
78\end{array}$ & & \\
\hline Squirt & 4.95 & 1.51 & 3. & 1.39 & 3.38 & .79 & 4. & 2.38 & & 5.65 & 1.38 & 4.32 & .40 & & $\begin{array}{r}.18 \\
87\end{array}$ & & \\
\hline Stamen & 8.08 & .99 & 2.39 & 1.51 & 2. & .90 & & 2.18 & & 7.61 & 1.42 & 2. & $\begin{array}{l}1.19 \\
1.39\end{array}$ & & $\begin{array}{l}.87 \\
89\end{array}$ & & \\
\hline Stance & 7.43 & 1.39 & 2.78 & 1.35 & 3.04 & .93 & 4. & 2.15 & & 2.15 & 1.35 & & $\begin{array}{l}1.39 \\
1.15\end{array}$ & & & & \\
\hline Staple & 536 & 1.45 & 4.38 & 1.08 & 3.88 & .90 & 4.50 & 1.84 & & & 1. & & $\begin{array}{l}1.15 \\
1.65\end{array}$ & & & & \\
\hline Starch & 6.08 & 1.38 & 3.25 & 1.44 & 4.00 & .75 & 5. & 2.11 & & & 1. & & $\begin{array}{l}1.65 \\
1.36\end{array}$ & & .87 & & \\
\hline Stench & 8.05 & 1.00 & 2.38 & 1.44 & 2.76 & .81 & 4. & 2.24 & & 7. & 1.53 & & $\begin{array}{l}1.36 \\
1.40\end{array}$ & & .77 & & \\
\hline ma & 8.24 & 1.12 & 1.91 & 1.30 & 3.27 & .81 & 2. & 1.7 & & 7. & 1.0 & 3 & & & .8 & & \\
\hline Strand & 6.60 & 1.50 & 3.23 & 1.44 & 3.39 & .86 & 4.46 & 1.8 & & & & & 1. & & .9 & 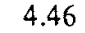 & 1.75 \\
\hline Stride & 6.81 & 1.17 & 3.23 & 1.20 & 3.33 & .76 & 4.81 & 2.1 & & & & & 1. & & .79 & & 2.25 \\
\hline Strife & 7.01 & 1.67 & 2.29 & 1.38 & 3.40 & .86 & 3.72 & 1.9 & & 8. & 1. & 1. & 1.34 & & .83 & 1. & 1.93 \\
\hline Studio & 6.82 & 1.31 & 3.91 & 1.14 & 3.79 & .79 & 5.88 & 2. & & 6. & 1.34 & 2 & 1.64 & & .85 & & 1.68 \\
\hline por & 8.21 & .93 & 2.42 & 1.41 & 3.08 & .99 & 3. & & & 7. & 1.41 & 1.59 & 1.03 & & .77 & & 1.77 \\
\hline way & 647 & 1.27 & 3.94 & 1.45 & 3.43 & .78 & 6 & 2. & & 8.3 & 1.12 & 1.55 & .95 & 2 & .71 & 1. & 2.04 \\
\hline Sucker & 4.73 & 2.11 & 3.26 & 1.55 & 3.75 & 81 & & & & 7.38 & 1.27 & 1.21 & 1.48 & $=$ & .92 & 4. & 1.61 \\
\hline Sugar & 2.31 & 1.32 & 4.86 & 1.19 & 4.40 & .6 & & & & 3.77 & 1.22 & 3.00 & 1.38 & 3. & .6 & 4. & 2.45 \\
\hline Suitor & 7.74 & 1.12 & 2.86 & 1.50 & 2.66 & .7 & & & & 5. & 1.06 & 3. & 1.4 & 3. & .85 & 5. & 1.60 \\
\hline Sultan & 7.80 & 1.44 & 3.06 & 1.73 & 2.32 & 6 & & 2. & & & & & 1. & & & & 2.06 \\
\hline Surtax & 8.44 & 1.14 & 1.41 & .95 & 2.36 & .0 & 2. & 2. & & 5.35 & 1.34 & 4.32 & 1.19 & 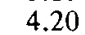 & .6 & 5. & 1.89 \\
\hline vey & 6.90 & 1.02 & 2.54 & 1.36 & 4.06 & .7 & & & & 5.64 & 1.77 & 3.29 & 1.65 & 3.21 & 1.09 & 4. & 1.37 \\
\hline the & 8 & 1.47 & 2.27 & 1.46 & 2.1 & .8 & & & & 4.80 & 1.43 & 4.62 & .88 & 3.7 & .7 & 6.14 & 2.24 \\
\hline abol & 6. & 1.23 & 2. & 1.60 & 4 & .7 & & & alrus & 5.96 & 1.52 & 4.65 & .7 & 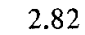 & .7 & 6.10 & 1.63 \\
\hline $\operatorname{tax}$ & & .99 & 1 & .8 & 2. & .9 & 2 & & arden & 7.32 & 1.07 & 3.73 & 1.25 & 3. & .88 & 4.37 & 1.86 \\
\hline Tailor & & 1.18 & 0 & 1. & & .7 & & & armth & 4.51 & 1.62 & 3.07 & 1.57 & 4. & .7 & & 2.02 \\
\hline Talcum & & 1.65 & & 1.37 & 3. & .8 & & $\begin{array}{l}2 \\
1\end{array}$ & ater & 1.54 & .67 & 4.79 & 1.38 & 4.8 & .6 & 7. & 2.17 \\
\hline Tangle & 5 & 1.61 & & 1.38 & 3.5 & .78 & 4. & & pon & 5.36 & 1.52 & 4.24 & 1. & & 80 & 9 & 1.83 \\
\hline Tango & 7.00 & 1.16 & 3.85 & 1.20 & 2.53 & .89 & 3.71 & 1. & & 5.01 & 1.49 & 3.40 & 1 & 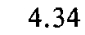 & .73 & 0 & 1.70 \\
\hline Tedium & 8.60 & .88 & 1.66 & 1.18 & 1.85 & .69 & 2.00 & 2. & & & 1. & 1 & 1. & ( & .89 & & 2.17 \\
\hline Theory & 7.13 & 1.19 & 1.84 & 1.19 & 4.29 & .83 & 4.62 & $\begin{array}{l}2 . \\
1 .\end{array}$ & Wintar & 3. & 1.55 & 4. & , & 4 & .5 & 1 & 2.05 \\
\hline Thorax & 8.33 & 1.07 & 2.63 & 1.4 & 2.51 & .96 & 3. & 2. & om & 6.37 & 1.24 & 1.90 & 1.3 & 3. & .6 & 9 & 1.87 \\
\hline Thread & 4.70 & 1.50 & 4.39 & 1.2 & 4.14 & .7 & 5. & 2. & Wither & 7.23 & 1.36 & 2.82 & 1.57 & 3.0 & .87 & 4.38 & 1.74 \\
\hline Throne & 5 & 1.46 & 4.24 & 1. & 2.5 & .7 & 5. & 2. & Wizard & 4.83 & 1.55 & 3.60 & 1.44 & 3.38 & .90 & 4.77 & 1.36 \\
\hline Throng & 7.36 & 1.58 & 2.60 & 1.5 & 2.69 & .8 & 3. & 2 & Wonder & 5.48 & 1.53 & 1.84 & 1.24 & 4.33 & .84 & 4.67 & 1.74 \\
\hline Ticker & 6.67 & 1.78 & 2.35 & 1.4 & 2.28 & .77 & 3.41 & $\begin{array}{l}2 . \\
1 .\end{array}$ & Wreath & 5.43 & 1.58 & 4.22 & 1.24 & 3.16 & .71 & 4.73 & 1.54 \\
\hline Tickle & 3.96 & 1.54 & 3.44 & 1.52 & 3.51 & .93 & 4.53 & 1. & Wrench & 6.64 & 1.57 & 3.77 & 1.56 & 3.3 & .93 & 4.92 & 1.79 \\
\hline Timber & 5.69 & 1.17 & 4.44 & .96 & 3.49 & 1.00 & 6.56 & & Wretch & 7.65 & 1.03 & 2.57 & 1.59 & 2.79 & .86 & 4.33 & 2.06 \\
\hline Tinder & 7.45 & 1.74 & 2.58 & 1.64 & 2.23 & .87 & 3.2 & & Yeoman & 8.23 & 1.05 & 2.48 & 1.45 & 2.35 & .76 & 3.31 & 2.83 \\
\hline Tingle & 5.62 & 1.53 & 2.5 & 1.5 & 3.43 & .83 & 4.33 & & Youth & 5.00 & 1.25 & 4.00 & 1.25 & 3.67 & .87 & 4.92 & 1.62 \\
\hline Tinker & 6.18 & 1.93 & 2.22 & 1.2 & 3.09 & .87 & 3.64 & & Zealot & 8.74 & .71 & 1.53 & 1.22 & 1.98 & .82 & 1.52 & 1.86 \\
\hline Tinkle & 4.82 & 1.89 & 3.18 & 1.52 & 3.22 & 1.00 & 3.14 & & Zenith & 7.96 & 1.48 & 2.72 & 1.50 & 3.12 & 1.05 & 3.80 & 2.06 \\
\hline Tinsel & 5.5 & 1.87 & 4.3 & 1.19 & 3.02 & .77 & 5.08 & $\begin{array}{l}1.4 \\
2.3\end{array}$ & & 8.62 & .92 & 1.77 & 1.21 & 1.8 & .73 & 1.28 & 1.72 \\
\hline Tirade & 8.60 & .85 & 1.49 & .94 & 2.27 & .89 & 1.96 & $\begin{array}{l}.50 \\
60\end{array}$ & Zodiac & 8.01 & 1.21 & 3.72 & 1.42 & 3.42 & .95 & 5.43 & 2.20 \\
\hline
\end{tabular}

